\title{
Testimonio de vida académica Entrevista de César Espinoza Claudio al profesor Zenón Eduardo Vargas Morales ${ }^{1}$
}

\author{
Artículos originales: ENTREVISTA \\ Recibido: 15/03/2021 \\ Aprobado: 25/06/2021 \\ Publicado: $14 / 10 / 2021$
}

1. Estimado Zenón, ¿puede usted hablarnos un poco sobre su trayectoria académica en San Marcos?

El año de 1967 ingresé a la Universidad Nacional Mayor de San Marcos para estudiar Sociología en la Facultad de Letras y Ciencias Humanas, cuando en el año anterior había egresado la cuarta promoción de sociólogos. Para esos años, era una carrera profesional totalmente desconocida en el Perú y en la mayoría de América Latina. Durante mi vida académica estudiantil tuve brillantes docentes de la Facultad de Letras, tanto en el ciclo de cultura general como en el de especialidad. Cómo olvidar a los docentes Javier Pulgar Vidal, Juan Ferro, Salazar Bondy, Luis Alberto Sánchez, Pablo Macera, Julio Cotler, José Russo, Washington Delgado, Aníbal Ísmodes, Sócrates Villar, José Matos Mar, son algunos nombres que recuerdo bien. Esta primera generación de docentes que enseñaban en la Escuela de Sociología fueron reemplazados por docentes jóvenes recién egresados, algunos de ellos con estudios de posgrado en FLACSO de Chile o en París. Entre

1 Licenciado en Sociología, con estudios de Economía, Maestría en Filosofía —-mención Epistemología-, por la Universidad Nacional Mayor de San Marcos. Estudios de Maestría en Administración de la Educación, por la Universidad Inca Garcilaso de la Vega; Diplomado en Pedagogía Universitaria por la Universidad de Lima; Diplomados en: «Gerencia Social y Gestión», «Políticas Públicas e Interculturalidad», "Políticas Públicas Gerencia y Gestión de Programas Sociales» por la Universidad Nacional Mayor de San Marcos; Posgrado en Periodismo de Investigación; Docencia Universitaria e Investigación por la Universidad Nacional Mayor de San Marcos. Ha sido catedrático principal e investigador de la Facultad de Ciencias Sociales, Director de la Escuela Académico Profesional de Sociología, Director Académico de la Facultad de Ciencias Sociales. Ha recibido diplomas de reconocimiento como profesor investigador por el Vicerrectorado de Investigación y como docente destacado por la Facultad de Ciencias Sociales, de la Universidad Nacional Mayor de San Marcos. los nuevos docentes del Departamento de Sociología estaban: César Germaná, Rodrigo Montoya, Walter Quinteros, Felipe Portocarrero, Aracelio Castillo, Roger Iziga, Víctor Medina, Fernando Lecaros, entre otros varios colegas. Posteriormente, en el año de 1975, ingresé a la docencia, incorporándome a esta segunda generación de docentes, no tan brillantes a comparación de quienes fueron mis maestros. Desde esa fecha, hasta que solicité mi cese en agosto de 2015, pasaron 40 años de trabajo permanente en la cátedra, con periodos en los cuales fue difícil ejercer la docencia por los contextos tan cambiantes que nos tocó vivir, como por ejemplo, el militarismo durante la década de 1970 , la presencia de la violencia terrorista durante la década de 1980, la intervención militar en la universidad durante la década de 1990, y finalmente, el retorno a los gobiernos democráticos en el presente siglo, donde los rectores todavía no logran realizar grandes cambios en lo académico, administrativo y la investigación. Son cuarenta años de aprendizaje en las aulas y de compartir la vida universitaria con jóvenes estudiantes principalmente de los sectores populares, quienes deseaban convertirse en buenos profesionales.

Durante mis primeros años de docente, dicté clases en la Escuela de Medicina, luego en las Facultades de Ciencias Económicas y Contables, Psicología, Filosofía y también en Ciencias Sociales. Desde 1985 en adelante, me dediqué exclusivamente a la docencia en la Escuela de Sociología, asumiendo el cargo de Director de la Escuela de Sociología en 1993 y después como Director Académico de la Facultad de Ciencias Sociales en tres oportunidades; además, participé activamente en los exámenes de admisión, en su 
organización y también en la elaboración de las pruebas. Una experiencia importante fue cumplir con la responsabilidad de organizar el examen de admisión en la ciudad de Tacna, con la participación de docentes de diversas facultades de la universidad. He sido responsable y miembro integrante de varios proyectos de investigación del Instituto de Investigaciones de la Facultad, cuyos resultados han sido publicados en las revistas académicas de la universidad como Estudios Sociales, Sociología e Investigaciones Sociales, y otros fueron empleados como documentos para el debate con los alumnos en las asignaturas que me tocó enseñar.

\section{2. ¿Usted empezó estudiando sociología y después ingresó a Filosofía? ¿Nos puede contar un poco acerca de su formación, siente que está relacionada con sus logros y proyectos académicos?}

Yo estudié sociología, después de concluir mis estudios, en 1973 postulé e ingresé a la carrera de economía en la Facultad de Ciencias Economía y Contables, ambas disciplinas se complementaron muy bien y fue de suma importancia para mi carrera docente, principalmente para cuando me tocó enseñar después algunos cursos en la Facultad de Ciencias Económicas y Contables y en la Escuela de Sociología. Mientras era estudiante de sociología leí algunas obras de Friedrich Von Hayek, quien decía que el que ha estudiado una sola profesión, es un mal profesional, por lo que era necesario tener amplios conocimientos para trabajar en una sociedad cada vez más compleja.

En los años de 1980 los alumnos recibían una formación ideologizada con el «marxismo", sin espacios para el debate en clase acerca de los trabajos de autores clásicos o fundadores de la sociología, descuidando también temas filosóficos y epistemológicos importantes. Cuando yo era estudiante, el profesor Salazar Bondy nos hizo leer "Los cuadernos filosóficos», una selección de textos de distintos autores que él mismo había hecho para sus alumnos. Recuerdo que me encantaba asistir a los conversatorios de los «viernes filosóficos» que organizaba la Escuela de Filosofía. A través de estos eventos, me di cuenta que las investigaciones en las ciencias sociales exigían un marco epistemológico que permita la demarcación entre la ciencia y la no ciencia. Tengo que confesar que estos eventos me abrieron los ojos, me di cuenta y reconocí que mis reflexiones teóricas y metodológicas tenían limitaciones, por lo que me encontré motivado en estudiar una maestría en filosofía, con mención en epistemología. Estoy agradecido con mis compañeros de aula por los debates que sostuvimos, algunos pertenecían a la Escuela de Filosofía de la Facultad de Letras de San Marcos, otros venían de universidades como la Católica (PUCP), de Ingeniería (UNI), la San Agustín de Arequipa (UNSA), y la San Antonio Abad del Cusco (UNSAAC). La mayoría de ellos tenían una formación en dos especialidades, lo que me permitió aprender de ellos diversos temas teóricos y epistemológicos, que en las clases ponían en apuros a los profesores, a pesar de que eran los más destacados de la Facultad de Letras: Arsenio Guzmán, Luis Silva Santisteban, Antonio Peña Cabrera, Luis Piscoya, Juan Abugattás, entre otros. Es a través de ellos que pude estudiar las diversas corrientes de la filosofía de la ciencia (epistemología) desde los Segundos Analíticos del libro de física de Aristóteles como parte de la filosofía clásica griega, pasando por Santo Tomás de Aquino en la época medieval, hasta llegar a la modernidad con la epistemología positivista y la concepción heredada (Círculo de Viena), con autores paradigmáticos como Karl Popper, Thomas Khun, Imre Lakatos, Kurt Gödel y Paul Feyerabend, además del marxismo. En general, estudiamos las diversas concepciones epistemológicas que sustentan las teorías de las ciencias sociales. Realicé varios trabajos de investigación: Epistemología genética, Historia de las Teorías de la Verdad, debates acerca del concepto de Paradigma en la obra de Thomas Khun, y Epistemología de las Ciencias Sociales, que fue mi trabajo para la tesis. El primer lugar de mi promoción lo ocupó un profesor de la PUCP que era economista y matemático, mientras que en mi caso logré modestamente el segundo lugar.

\section{Como integrante de la segunda generación docente de sociología en San Marcos, usted ha vivido acontecimientos importantes como la caída $y$ muerte de Mao Tse Tung, además del triunfo del Neoliberalismo en el mundo. ¿Considera que estos grandes sucesos mundiales generaron impactos y reflexiones en las Ciencias Sociales dentro y fuera de San Marcos?}

Lo que recuerdo de mis años de estudiante y docente es el mes de octubre, bautizado por la voz de los alumnos como el «octubre rojo». Era el mes en el que 
se celebraban aniversarios de acontecimientos como la revolución china de 1949 y la revolución bolchevique de 1917. Era un mes donde los dirigentes estudiantiles organizaban charlas, seminarios, celebraciones, ya sea desde las posturas políticas que en ese tiempo se les denominaba pro-chinos o pro-soviéticos. Cuando era alumno de la Escuela, si algún profesor mencionaba estos acontecimientos lo hacía sin darle mayor importancia; en cambio los dirigentes y líderes de la vertiente izquierdista conocían al detalle estos hechos históricos. Recién cuando ingreso a la docencia en el año de 1975, encuentro que la mayoría de los alumnos estaban totalmente ideologizados con sus respectivos «santos» (como le gustaba decir al profesor Julio Cotler). Había trotskistas, stalinistas, guevaristas, maoístas, albaneses, etc. Fue a través de ellos que tuve la ocasión de leer algunos trabajos de Mao, como "Las investigaciones en Junán», «las clases sociales en China", "las cuatro tesis filosóficas", entre otros textos que eran de conocimiento de los alumnos. Definitivamente, fueron años de mucho debate acerca de la sociedad peruana, como ocurría al abordar el carácter del gobierno militar y el «carácter de la sociedad peruana». Aquí la tesis doctoral de Rodrigo Montoya: «sobre el carácter predominantemente capitalista de la economía peruana» se convirtió en un texto de referencia entre los que sostenían el «carácter feudal o semifeudal» o "capitalista» de la sociedad peruana. Los alumnos resaltaban los hechos más importantes de Mao como «el gran salto adelante» con la colectivización de la agricultura, y luego la «Revolución cultural» (1966) que duró hasta su muerte en 1976. En 1978, Deng Xiaoping lidera una serie de reformas, bajo la experiencia de haber vivido en la Europa capitalista, abrió la entrada a los capitalistas extranjeros y a la inversión. A partir de esa fecha, y con la caída del muro de Berlín en 1989, todas esas diversas experiencias, orientadas a construir una sociedad diferente al capitalismo y el liberalismo, fracasaron. Hoy en día las dos grandes potencias, China y Rusia (la antigua URSS), tienen ahora dirigentes que asumen la economía de mercado y la privatización con algunas diferencias. Para los años ochenta están Margaret Thatcher en Gran Bretaña y Ronald Reagan en Estados Unidos, consolidando lo que se conoce ahora como la economía neoliberal.

Me viene a la memoria el debate realizado en la maestría de filosofía con el profesor Juan Abugattás, quien nos planteó si el liberalismo y neoliberalismo acaso eran ideologías, corrientes filosóficas, pensamientos económicos, o qué más, para finalmente explicarnos que el liberalismo era un fenómeno que venía desde la revolución francesa. Hizo referencia a autores liberales como Adam Smith, David Hume, Jeremy Bentham, John Stuart Mill, Von Hayek, y Friedman. Que, en resumidas cuentas, el pensamiento liberal consistía en una concepción y actitud individualista, ya sea acerca del Estado, la sociedad, $y$, principalmente, la economía y el derecho, con igualdad ante la ley y no igualdad de oportunidades para todos. Como conclusión, diría que después de la constitución de 1993 y la dictadura fujimorista, nos encontramos con un neoliberalismo radical en lo económico, con un papel subsidiario del Estado, cuyos resultados son los que estamos viviendo hoy en día con el azote de la pandemia: instituciones colapsadas en la salud y la educación. Hoy en día hasta los mismos defensores del neoliberalismo exigen una mejor atención del Estado, después de promover durante décadas que el bienestar humano se alcanza a través de las capacidades individuales y empresariales en un marco del libre mercado, libre comercio y libre competencia, donde el papel del Estado debe ser mínimo y subsidiario para garantizar el cumplimiento de estas condiciones.

4. Yo recuerdo que usted en los años 70 se emocionaba mucho con la enseñanza de los temas rurales, le gustaba practicar los trabajos de campo e impulsar la difusión de las observaciones, hallazgos e investigaciones de los alumnos y docentes como primeras reflexiones sobre los cambios que estaban ocurriendo en el país en los años en que terminaba el gobierno militar. ¿Puede usted explicarnos qué tipo de metodología usaba para el proceso de desarrollo y redacción de un proyecto, en la perspectiva de los estudios de caso?

El hecho de haber vivido mi infancia en un centro poblado rural confirmó mi interés por los temas de la sociología rural. En los años de la década de 1970, hubo grandes cambios después de la reforma agraria (1969) con Juan Velasco Alvarado, quien marcó un punto de inflexión en la historia política peruana. Se rompe con la estructura del poder oligárquico, las grandes haciendas fueron adjudicadas a sus trabajadores, convirtiéndose en Cooperativas Agrarias 
de Producción, y los latifundios serranos fueron la base para la formación de los Sistemas Agrarias de Integración Social (SAIS). Paralelamente se emprende la reforma educativa bajo la conducción de quien fue nuestro profesor, Augusto Salazar Bondy, en su condición de Ministro de Educación. También se realizó una campaña de alfabetización a nivel nacional acompañada de la enseńanza y reconocimiento del quechua como idioma oficial. En 1971, crearon el Sistema Nacional de Apoyo a la Movilización Social (SINAMOS), con la finalidad de organizar políticamente a los diversos sectores sociales y así contar con el apoyo para los grandes cambios en curso.

Eran ańos donde se vivieron profundos cambios en la estructura económica, social y política. Pero estos cambios, para la mayoría de alumnos, eran vistos a la distancia solo desde las aulas, motivo por el cual, en mi condición de docente responsable en asignaturas de estructura agraria y seminarios de investigación rural, siempre consideré que los debates en el aula eran importantes para analizar estos procesos de cambios, pero los alumnos tenían que formarse con experiencias de contacto directo con la realidad, no sólo geográfica, sino también humana, social, conversando con las autoridades locales, los dirigentes, la población en general y así conocer mejor, entender de cerca sus principales problemas, conflictos, potencialidades, limitaciones, en distintas áreas, como su organización política, sus modos de vida, su historia, etc. Todo esto era posible solo con las salidas al campo, previo estudio bibliográfico acerca del lugar donde se iba realizar la práctica de campo y una revisión de los datos disponibles en el Instituto Nacional de Estadística.

Cuando planeábamos viajar a la zona del Callejón de Conchucos, en la provincia de Huari, revisamos los viajes de Santo Toribio de Mogrovejo a fines del siglo XVI, quien hizo el recorrido montado en mula por la sierra de Cajamarca, Pasco y Ancash. Esta tarea tenía como finalidad que los alumnos se sientan más preparados y con menos temor de hacer un viaje a las alturas de la sierra. Este viaje lo planificamos a través de un proyecto de investigación, un documento que contenía objetivos precisos, un método de trabajo con observaciones, entrevistas, historias de vida, reuniones con autoridades, dirigentes y campesinos, además de un cronograma con actividades día por día. El documento se presentaba al decano de la Facultad para su aprobación por el Consejo de Facultad, luego el decanato emitía una resolución que respaldaba el trabajo de campo frente a las autoridades locales y policiales. Por otro lado, los alumnos organizaban algunas actividades de apoyo a sus compañeros que no tuvieran los recursos económicos para los gastos diarios en el campo. En algunas ocasiones recibimos el apoyo económico de la Facultad, e incluso algunas ONGs nos brindaban dicho apoyo a cambio de compartir los resultados del trabajo de campo. Fueron muchos trabajos de campo, como las salidas al valle de Ica, y los recorridos por Hierro Perú; el viaje al Alto Piura para estudiar la situación de 11 cooperativas, con el apoyo del Centro de Investigación y Promoción del Campesinado (CIPCA) dirigido por el padre Vicente Santuc; también fuimos a Chucarapi en Arequipa, donde nos recibió el alcalde José Villalobos Ampuero de Izquierda Unida (1981-1983); estudiamos las cooperativas y sus conflictos en el Valle de Huaura, Huacho; la visita a la comunidad campesina de Cátac (Ancash) para estudiar las relaciones y los conflictos entre la hacienda Utcuyacu, los campesinos de Cátac y las empresas mineras; las excursiones al Callejón de Conchucos, principalmente a los distritos de la provincia de Huari, donde el alcalde convocó a las principales autoridades como los regidores, el director de UGEL, el Fiscal, los jefes de salud y la policía.

En estas salidas, los alumnos recién conocían de forma directa cómo se hacían gestiones para el desarrollo local o microregional, se acercaban a las comunidades campesinas no sólo en su organización, también tenían contacto directo con varias situaciones de pobreza rural y falta de presencia del Estado. Aprendieron a compartir, a conocer la pobreza de los campesinos. Los alumnos me contaban que en algunas visitas tuvieron que sentarse junto al fogón para almorzar.

Por nuestra condición de sanmarquinos, siempre fuimos bienvenidos por las autoridades y la población. Por ejemplo, en Piura, el profesor Távara, alcalde del distrito de Chulucanas, nos declaró «visitantes ilustres» cuando fuimos presentados a los alumnos de un colegio. En Ancash, la Confederación Campesina del Perú realizaba su Congreso Nacional en la comunidad campesina Carhuayoc del distrito de San Marcos, y fuimos invitados para participar en las discusiones acerca de la problemática agraria y rural de la zona. Al regresar a las aulas, nuevamente debatíamos 
acerca de los diversos temas y problemas presentados, pero esta vez incluyendo el conocimiento empírico y la experiencia de cada uno en nuestras prácticas de campo. Luego de sistematizar, analizar y redactar los informes, se sustentaban en un Seminario más amplio, que era organizado por todos los seminarios de las Escuelas de la Facultad para compartir las diversas experiencias de investigación. Para mí, como docente, todo esto fue una experiencia inolvidable, me permitió conocer, aprender y compartir los aciertos, las inseguridades y los logros de los alumnos en su formación profesional. Quizás por todas estas experiencias, mis alumnos me reconocen y saludan con cariño cuando voy por la calle o la universidad, con algunos mantengo una amistad profesional y conversamos seguido, ahora veo que son personas adultas avanzando por la vida. En una ocasión, una de mis exalumnas estaba caminando con sus hijos y su esposo, se acercó para saludar y darme un abrazo, me dijo: «Profesor, sus alumnos nunca nos olvidaremos de usted. Yo le tengo mucho aprecio, a pesar de que me desaprobó en su curso, pero fue por mi culpa». Cuando he viajado al extranjero, siempre se me presentó la oportunidad de encontrarme con mis exalumnos en diferentes lugares, donde radican o ya tienen una familia, demostrando aprecio. El cariño de mis alumnos es lo más gratificante en mi condición de docente.

\section{5. ¿Puede contarnos sobre la producción intelectual que lo animaba en esos años, el tipo de libros y otras publicaciones que consultaba, y qué resultados de su labor como docente lograron finalmente difundirse dentro o fuera de San Marcos?}

Cuando ingreso a la docencia en 1975, me asignaron el curso de Seminario Rural, como asistente del profesor principal. La reforma agraria y otros cambios realizados por los militares en el régimen de Juan Velasco originaron los más encendidos debates, no solo en la universidad, sino también en la sociedad civil, entre los dirigentes políticos, las organizaciones campesinas, y, principalmente, entre los profesionales de las ciencias sociales y afines: sociólogos, antropólogos, historiadores, economistas, abogados, agrónomos, politólogos, y algunos ingenieros. En cuanto a tu pregunta acerca de los libros, entre los de lectura obligatoria estaban Los 7 ensayos de interpretación de la realidad peruana de José Carlos Mariátegui, Los dueños del Perú de Carlos Malpica, Horas de lucha y Páginas libres de Manuel Gonzáles Prada, Las venas abiertas de América Latina de Eduardo Galeano -que luego se arrepintió de su trabajo-, Nacionalismo, Neoimperialismo y Militarismo de Aníbal Quijano, y dos libros de Rodrigo Montoya: Capitalismo y no capitalismo y A propósito del carácter predominantemente capitalista de la economía peruana. Además, los docentes e investigadores de la Universidad de San Marcos, la Católica, el Instituto de Estudios Peruanos, CEPES, DESCO, publicaban un conjunto de trabajos principalmente acerca de temas agrarios y rurales.

Entre los libros de consulta teórica, estaba El Capital de Marx, El desarrollo del capitalismo en Rusia y las Obras completas de Lenin, Kautsky, y varias publicaciones de la editorial Siglo XXI. Por esa época aparecieron muchas revistas de izquierda como: Sociedad y Politica, Socialismo y Participación, Quehacer, Marka, Debates en sociología, Apuntes. Luego, a mediados de los años ochenta, se publica El zorro de abajo y Márgenes, donde varios intelectuales de izquierda debatían acerca de la problemática nacional y de América Latina. En cuanto a las investigaciones realizadas en la Facultad de Ciencias Sociales, diría que el trabajo realizado fue más individual que colectivo o institucional. Eran ańos en que aún no existía la Unidad de Posgrado y el Instituto de Investigaciones recién empezaba a organizarse bajo la dirección del Dr. César Fonseca, con quien realizamos un proyecto de investigación en Junín como parte de un equipo de docentes y egresados de la Facultad. Cada profesor tenía sus propias aspiraciones temáticas, sin mayores resultados grupales. Posiblemente una excepción a esto fue el proyecto de investigación que impulsó el Dr. Aracelio Castillo con un grupo de docentes de la Facultad, con la finalidad de estudiar histórica y sociológicamente la «Estructura agraria del valle de Piura», el cual lamentablemente quedó frustrado por discrepancias políticas e ideológicas. Cuando me incorporo a este proyecto, me encontré con Alicia Suárez, quien recién egresaba y tuvo un breve tiempo de participación. En cambio, para mi caso, como docente, continué en el proyecto, primero acopiando información en el Archivo del Fuero Agrario que quedaba en el distrito del Rímac, luego viajé a Piura en dos oportunidades para recoger datos, entrevistar a dirigentes, funcionarios y docentes. Luego la información fue sistemati- 
zada, elaboramos un conjunto de cuadros y gráficos en papel milimetrado, y la redacción estuvo a cargo del profesor Aracelio, quien lamentablemente fallece en diciembre de 1977, dejando inconcluso el trabajo de investigación. Sin embargo, ya habíamos publicado los resultados preliminares, los cuales fueron entregados al Instituto de Investigaciones y también presentados en diversos seminarios de investigación, para finalmente publicarse en la revista Estudios que editamos hasta un tercer número.

\section{En los años de 1990 ya lo escuchábamos} comentar y plantear asuntos sobre el tema de la epistemología. ¿Podría usted explicarnos cómo es que la epistemología puede auxiliar a los investigadores en el análisis de las fuentes sociológicas?

Quisiera empezar señalando que existen varias formas de abordar la investigación en ciencias sociales. Cuando se pretende hacer ciencia sociológica, histórica, económica, antropológica, ya sea con procedimientos mecanicistas y matemáticos, acentuando la relevancia de las leyes generales para la explicación científica, entonces estamos dentro de la fundamentación epistemológica del positivismo. Pero, por otro lado, existe una tradición en el uso de métodos para la comprensión e interpretación, donde el enfoque es de carácter hermenéutico, considerado en algunos casos como menos instrumental en el proceso del conocimiento científico. Hay que tener en cuenta que estos dos enfoques en la investigación han sido protagonistas de un falso debate epistemológico en las ciencias sociales. Hoy en día se está superando este debate a través de una triangulación teórica, metodológica y procedimental, entendiendo la investigación como un proceso que involucra la observación, descripción, comprensión y explicación del problema social que va a estudiarse.

En ese sentido, la epistemología nos permite reflexionar y evaluar diversos aspectos del proceso del conocimiento científico con la finalidad de validar sus resultados. Para la fundamentación epistemológica de la investigación en ciencias sociales, han sido sumamente valiosos los debates acerca del empirismo, positivismo, neopositivismo, el racionalismo crítico de Karl Popper, la estructura de la revolución científica de Thomas Khun y el debate entre Lakatos y Feyeraben, sin dejar de lado enfoques como los del posmarxismo y la teoría crítica de Frankfurt. En el campo metodológico, estos enfoques continúan generando debates y coexisten con propuestas epistemológicas contemporáneas tales como los sistemas complejos de Edgar Morin, además de la fenomenología, la hermenéutica y el constructivismo en las ciencias sociales.

Las reflexiones epistemológicas tienen una larga historia, desde Aristóteles hasta la actualidad, pero es recién en el siglo XX que adquieren una mayor significación y relevancia, especialmente cuando tenemos dos enfoques epistemológicos conocidos como las «ciencias duras» y las "ciencias blandas», que configuran dos grandes conjuntos de maneras de analizar y comprender el mundo. Por ejemplo, para la concepción heredada, la lógica y la fundamentación empírica son los únicos elementos que justifican la validez de las teorías científicas. Según la epistemología neopositivista, la objetividad en el conocimiento científico es validada de acuerdo con una estructura lógico-conceptual, con la unidad de la ciencia, basándose en evidencia empírica. En otra postura epistemológica, la atención está centrada en el tema de la comprensión y la explicación, aplicando métodos interpretativos como nos sugería Max Weber, considerando varias propuestas como el interaccionismo simbólico, la etnografía, la etnometodología, y la teoría fundamentada. Cada una de estas propuestas se diferencian por las formas de trabajo metodológico que requieren según el tipo de problema que ocupa la atención del investigador. Por ejemplo, desde un enfoque weberiano, lo que interesa en la investigación es el entendimiento interpretativo de la acción social, a través de la explicación de sus causas y sus consecuencias. En conclusión, la epistemología nos permite examinar las fronteras del conocimiento con el objetivo de generar conocimientos universalmente válidos.

\section{7. ¿Nos puede contar usted acerca de cómo} interactuó con distintas orientaciones teóricas en sus trabajos de investigación? ¿Pasó del marxismo hacia el estructural-funcionalismo, o fue al revés?

Yo estudié a fines de la década de 1960, personalmente, tuve la experiencia en tener profesores positivistas, estructural-funcionalistas, y otros que se iniciaban en el análisis de la realidad social desde la perspectiva de Marx, principalmente, desde la teoría crítica de la escuela de Frankfurt. Me acuerdo de los profeso- 
res en cultura general y especialidad como Aníbal Ísmodes, José Mejía Valera, Román de Silgado, Walter Quinteros, quienes tenían un enfoque positivista en el análisis de los temas sociales. También estaba Julio Cotler, que regresaba de la universidad de Burdeos con una visión más abierta en sus análisis; Rodrigo Montoya que regresaba de su doctorado en París, quien en el curso de Estructura Social nos explicó cada uno de los temas desde la perspectiva de Marx; Felipe Portocarrero, quien nos enseñó la parte metodológica del libro de Lenin El desarrollo del capitalismo en Rusia; César Germaná, que enseñaba el curso de sociología política con toda la influencia del marxismo francés. Leímos La imaginación sociológica de Wright Mills, también a autores que desarrollaban varios temas - por ejemplo, la teoría de la dependencia- como Poutlanzas, Althusser, Cerroni. Cuando ingreso a la docencia como auxiliar en la cátedra de Seminario de Investigación Rural asignada al Dr. Aracelio Castillo, yo había asimilado la «Teoría de los modos de producción" para estudiar la estructura social de Piura, un conjunto de conceptos planteados por Marx y Engels en 1845. Consistía en estudiar la organización de la actividad económica, apoyándonos en las características históricas y sociológicas de sus organizaciones, las formas de participación económica de los trabajadores, las interacciones sostenidas al interior de dicho grupo y con la sociedad en su conjunto. Personalmente, tanto en las clases con mis alumnos, como también en mis investigaciones, siempre he tenido una perspectiva abierta en términos teórico-metodológicos, según el tema o problema que me preocupaba estudiar.

\section{En San Marcos se conoce de manera general que los colegas sociólogos Julio Cotler, Aníbal} Quijano, Ismodes Cairo y Aracelio Castillo usaban el marxismo como un marco para la lectura de los cambios y las continuidades sociales. ¿Puede usted señalarnos cómo fueron utilizados el marxismo y otras reflexiones sociológicas para ofrecer una visión general y estructural del Perú de los 80 s?

El profesor Aracelio fallece en 1977, por lo tanto, no fue testigo de las cosas que ocurrieron en los ańos de 1980. Como he explicado en la pregunta anterior, el profesor enfocó sus investigaciones y análisis de los problemas nacionales a través del marxismo, pero no un marxismo de manuales o panfletos, sino como re- sultado de una lectura directa de los escritos de Karl Marx como Los Grundrisse y El Capital, dándole importancia a la «Teoría de los modos de producción». En el caso de Aníbal Ísmodes, no se aprecia algún trabajo de investigación, sino algunos textos teóricos, como su libro clásico Sociología, donde analiza los principales conceptos sociológicos como: valores sociales, movilidad social, cambio social, control social, la familia, la religión, las instituciones, la desviación social, etc., desde un enfoque positivista y estructural-funcionalista. Para el caso de Julio Cotler, quien fallece en 2019, sus investigaciones giran en torno a las cuestiones política del país y de América Latina: la educación, los partidos políticos, el Estado, los movimientos sociales, la democracia entre otros temas, estos temas los estudió desde la perspectiva de la sociología histórica comparada. En sus análisis confluyen diversas perspectivas y enfoques, en ese sentido, en sus obras uno puede encontrar influencias de Karl Marx, de Weber, así como el estructural funcionalismo, cuando analiza los conflictos sociales.

Finalmente, en cuanto a Aníbal Quijano, quien falleció con 90 años cumplidos, el 31 de mayo de 2018, sus obras publicadas tienen una gran influencia del enfoque marxista, como «Crisis imperialista y clase obrera en América Latina», en el que plantea un análisis desde las clases sociales, pero en sus trabajos publicados más recientes prefiere utilizar los conceptos de eurocentrismo, colonialidad, poder, raza, dominación y explotación. Es importante destacar su tesis de que el elemento constitutivo del poder capitalista se funda en la clasificación racial étnica de la población. La trayectoria de Aníbal nos dejó un legado de muchos artículos y libros como Nacionalismo, neoimperialismo y militarismo en el Perú, Crisis imperialista y la clase obrera en América Latina, Imperialismo y marginalidad en América Latina, Dominación y cultu$r a$, donde analiza, desde su preocupación intelectual, diversos temas acerca de cómo evoluciona la política en el Perú, como lo cholo y el conflicto cultural, la colonialidad y descolonialidad del poder, lo racial y la clasificación social, que tienen mucho debate no solo en el Perú, sino en toda América Latina.
9. Otra experiencia académica e institucional que vivió fue su participación como autoridad en el Colegio de Sociólogos del Perú. A grandes rasgos, ¿Nos puede comentar qué tipo de experiencias 
y reflexiones tuvieron prioridad? ¿Qué avances se lograron en el proceso de construcción de una identidad del sociólogo peruano y sanmarquino?

El Colegio de Sociólogos del Perú fue creado en el año de 1989, y se formó una Junta transitoria con la finalidad de elaborar el estatuto y organizar la elección de la primera Junta directiva en 1993. Tuve el honor de formar parte de la primera junta directiva con destacados sociólogos como el Dr. Henry Pease García, elegido como Decano Nacional, y el Dr. Aníbal Ísmodes Cairo, como el Decano Regional para Lima y el Callao. Esto ocurre durante el gobierno de Alberto Fujimori en el que se instala el Congreso Constituyente Democrático para la elaboración de la nueva Constitución del Perú. El Dr. Henry Pease, a pesar de sus múltiples ocupaciones congresales, se dio tiempo para organizar el Colegio y así poder cumplir con los objetivos trazados del programa para las elecciones. Pero en el ańo 1994, solicitó licencia por un año, motivo por el cual, en mi condición de Secretario Nacional, asumí la encargatura del decanato, debido a que el Vicedecano también era el Decano Regional de Cajamarca y trabajaba en la Universidad Nacional de Cajamarca. Los ingresos económicos generados por la inscripción de los colegiados no cubrían los gastos fijos como el alquiler del local y los pagos a la secretaria. Para poder cubrir estos gastos, cada uno de los miembros de la Junta Directiva aportábamos mensualmente una parte de la cotización, siendo el Dr. Pease quien aportó una mayor proporción. Gracias a la efectiva participación de la Junta Directiva, logramos desarrollar un conjunto de actividades, como la defensa de la profesión de acuerdo con las normas vigentes y el estatuto del Colegio, fue una tarea difícil, pues en muchas instituciones académicas los cursos de sociología estaban a cargo de otros profesionales. Se realizó un seminario en el que participaron los directores de Escuela de todas las universidades donde se formaran profesionales sociólogos, con la finalidad de discutir y debatir las experiencias en cuanto a la malla curricular y la enseñanza de esta profesión. También se organizó otro seminario con motivo del nacimiento de José Carlos Mariátegui, con la participación de destacados especialistas en el tema como Aníbal Quijano, Manuel Marzal y Rodrigo Montoya. Realizamos varios seminarios más, de actualización profesional, con la participación de los docentes de la Pontificia
Universidad Católica del Perú y de la Universidad Nacional Mayor de San Marcos.

Formamos varios grupos de trabajo con la finalidad de debatir diversos temas de acuerdo con la especialidad de cada uno de los participantes miembros de la Orden. Establecimos una colaboración permanente con la Junta Directiva de los Colegios Regionales. Fueron dos ańos de trabajo intensos en los que sentamos las bases para el buen funcionamiento de la institución. Convocamos a la elección de la nueva Junta Directiva, donde fue elegido Decano Nacional el Dr. Sinesio López Jiménez, docente de San Marcos. Posteriormente, durante la decanatura de la Dra. Narda Henríquez, fui su vicedecano nacional, en ese periodo realizamos diversas actividades, cursos de actualización con la finalidad de recaudar fondos para el Colegio, e iniciamos la publicación del boletín del Colegio. También tuve el honor de integrar la directiva del Dr. Denis Sulmont cuando fue elegido Decano del Colegio Regional Lima, responsabilizándome de la secretaría de asuntos científicos y culturales.

\section{Finalmente, ¿Puede usted hablarnos sobre los temas a los que se dedica ahora y qué tipo de investigaciones está realizando?}

Hace cuatro años dejé la universidad en mi condición de docente, pero en los siguientes dos años me invitaron para dictar clases en el curso de actualización para jóvenes egresados de la Escuela de Sociología, lo que me permitió retomar el contacto con los alumnos jóvenes, así como estar más pendiente de nuevas publicaciones teóricas, de investigaciones aplicadas y revistas académicas. Siento que no me afectó mucho el hecho del alejamiento de las aulas después de 40 años de convivencia con los alumnos, no solo en los salones de clase, sino también en los pasadizos y el cafetín de la Facultad. Al dejar la universidad, pude retomar algunos trabajos de investigación que estaban inconclusos, lo que me ha permitido también ordenar muchos datos sueltos que había recogido en los trabajos de campo, para su discusión con los alumnos en el aula. Uno de estos trabajos es acerca de la zona donde nací, "Huari Subregión Puchka: memoria histórica entre la descentralización, la gobernabilidad y las relaciones de corrupción (minería, pobreza y desarrollo local)», donde trato de explicar los impactos generados por la empresa minera Antamina, y los comportamientos de las autoridades locales en 
cuanto a la gestión del canon minero y los conflictos sociales.

Otro trabajo que está concluido es acerca de la «Universidad, sociedad y sociología en tiempos de conflicto", en el que, principalmente, hago una memoria de mi experiencia personal como alumno y luego como docente desde los ańos de 1960 hasta el momento en que se escribe el libro. Analizo la reflexión sociológica y los hechos sociales y políticos que ocurren durante el periodo del militarismo con Juan Velasco Alvarado; la década de 1980, con el rol de la izquierda y la presencia de grupos terroristas como Sendero Luminoso y el MRTA; los años de 1990 con el gobierno Fujimori y la intervención en la universidad; para luego concluir con todos los hechos relevantes que se vivieron durante el presente siglo.

Otro trabajo concluido es lo que había iniciado como tesis para la maestría en filosofía sobre «La epistemología de las ciencias sociales», que analiza los diversos enfoques epistemológicos y metodológicos en la investigación social. Del mismo modo, voy desarrollando una investigación llamada «La vida social, académica y política del estudiante universitario», un estudio de caso de alumnos en San Marcos. Otro texto más trata acerca del pueblo rural donde nací, que pertenece al distrito y provincia de Huari, "Mallas: un pueblo de emprendedores», en el que investigo el origen de sus principales familias, así como la organización social del pueblo y sus costumbres, destacando la situación social y el emprendimiento económico de los migrantes radicados en la ciudad de Lima, que han logrado realizarse como profesionales en diversas especialidades y también como pequeños empresarios que han alcanzado reconocimiento institucional en sus rubros.

Finalmente, estoy escribiendo un libro acerca de "Las etapas de la sociología y sus representantes», una sistematización del pensamiento social, desde sus inicios con la filosofía clásica griega, hasta las nuevas sociologías actuales, pasando por los clásicos y los posclásicos. Estos trabajos están casi concluidos, es posible que se publiquen virtualmente porque no estoy en la posibilidad de hacer ediciones impresas por los costos elevados.

\section{Gracias por la entrevista.}

Febrero, 2021. 